\title{
A 42-year-old man with elevated ferritin
}

\author{
Matthew B. Lanktree MD PhD, Bekim Sadikovic PhD, Mark A. Crowther MD MSc
}

Competing interests: None declared.

This article has been peer reviewed.

Correspondence to: Matthew Lanktree, matthew.lanktree @ medportal.ca

CMAJ 2015. DOI: 10.1503 /cmaj.140259

\begin{abstract}
A 42-year-old businessman of South Asian descent presents with a prolonged history of fatigue and bothersome joint pain in his hands and wrists. The patient takes metformin for type 2 diabetes and ramipril for hypertension. He consumes 3 martinis per night and does not smoke. A physical examination shows no active joints, and his abdomen is normal with no evidence of chronic liver disease. Previous laboratory investigations showed elevated ferritin (660 [normal < 300] $\mu \mathrm{g} / \mathrm{L})$ and hemoglobin (178 [normal 115-165] g/L), and normal liver enzyme levels.
\end{abstract}

\section{What is the most likely diagnosis?}

Alcoholic and nonalcoholic fatty liver disease are the most likely causes for the patient's elevated ferritin, but hereditary hemochromatosis is also possible. Hereditary hemochromatosis is a genetic condition in which elevated iron stores lead to iron deposition in the liver, heart, pancreas, skin, joints, pituitary gland and testis, resulting in cirrhosis of the liver, heart failure, diabetes, skin bronzing, arthritis, endocrine abnormalities and cancer. ${ }^{1}$ However, the classic "bronze diabetes" presentation is uncommon. ${ }^{1}$

\section{What other diagnoses should be considered?}

Acute and chronic inflammation resulting from infection, malignant growths, renal failure, liver disease, metabolic syndrome and autoimmune

\section{Box 1: Summary of recommendations from current clinical} guidelines ${ }^{3,4}$

- Genetic testing should not be used to screen for hemochromatosis.

- Patients with elevated transferrin saturation (>0.45\%) and ferritin $(>300 \mu \mathrm{g} / \mathrm{L}$ ) should undergo testing for the C282Y and H63D alleles of the HFE gene.

- Phlebotomy should be performed in patients with elevated transferrin saturation and ferritin who have a positive result on genetic testing.

- Patients who show evidence of iron overload, but who do not have a positive result on genetic testing, should still be considered for phlebotomy or liver biopsy if no other cause is apparent.

- Elevated ferritin is very sensitive, but not specific, for iron-related liver damage. conditions may lead to elevated ferritin. ${ }^{2}$ Environmental exposures including diet, alcohol and medications, particularly iron administered orally or intravenously, can also elevate ferritin and increase the risk of pathological iron accumulation. Elevated ferritin in the context of elevated transferrin saturation suggests iron accumulation. $^{2}$

\section{What investigations should be done?}

A detailed history should be taken and a physical examination should be performed. In addition, iron stores should be assessed by measuring transferrin saturation, and renal function should be assessed by measuring serum creatinine. Chronic hepatitis infection and inflammation can be ruled out by serology and by determining the erythrocyte sedimentation rate, respectively. The patient should undergo investigations to assess for metabolic syndrome, including glycated hemoglobin, fasting glucose and a lipid profile. Finally, liver ultrasonography should be done to determine whether nonalcoholic fatty liver disease and cirrhosis are present. ${ }^{2}$

\section{Should the patient undergo genetic testing for hemochromatosis?}

Genetic testing should not be used to screen for hemochromatosis, but current guidelines ${ }^{3,4}$ suggest testing in patients with elevated transferrin saturation (>0.45\%) and ferritin (> $300 \mu \mathrm{g} / \mathrm{L})$ (Box 1). Testing typically involves genotyping two polymorphisms, C282Y and H63D, in the hemochromatosis gene (HFE). A positive result is defined as two copies (homozygosity) of the C282Y allele, or a C282Y allele on one chromosome and an H63D allele on the other chromosome (compound heterozygosity). ${ }^{5}$ Less than $0.5 \%$ of people with European ancestry are homozygous for $\mathrm{C} 282 \mathrm{Y}$, with even lower prevalences in other ethnicities. ${ }^{5}$ Although C282Y homozygosity is found in about $80 \%$ of patients with clinical hemochromatosis, iron overload will not develop in $60 \%-80 \%$ of patients with a positive test result. ${ }^{3}$ Other genetic causes of hemochromatosis have been described, and iron 
overload may still develop in patients with a negative result on standard HFE testing, particularly if the patient is not of European descent. ${ }^{3}$

\section{What is this patient's prognosis?}

In the presence or absence of a positive result on $H F E$ testing, patients with biochemical evidence of iron overload are at risk of cirrhosis of the liver, cardiomyopathies, arthropathies and endocrinopathies resulting from the accumulation of nontransferrin-bound iron. Hemochromatosisrelated cirrhosis typically occurs in patients with ferritin levels higher than $1000 \mu \mathrm{g} / \mathrm{L} .{ }^{3,4}$ Less than $10 \%$ of patients with $H F E$ hemochromatosis show end-organ manifestations. ${ }^{4}$

\section{Are further investigations required if hemochromatosis is diagnosed?}

In cases of hemochromatosis with a ferritin level higher than $1000 \mu \mathrm{g} / \mathrm{L}$, a biopsy of the liver should be considered. ${ }^{3}$ Initial noninvasive investigation with transient elastography can identify advanced fibrosis and cirrhosis, although access to such imaging may be limited in smaller centres. ${ }^{3}$ In patients with hemochromatosis-induced cirrhosis of the liver, ultrasound surveillance for hepatocellular carcinoma should be performed every 6 to 12 months. ${ }^{4}$ Because cardiac involvement is unusual, electrocardiography and echocardiography are reserved for patients with clinical indications. ${ }^{3}$ Radiological evaluation of affected joints is appropriate. ${ }^{3}$

\section{Does this patient require phlebotomy?}

Phlebotomy is a safe procedure that is both therapeutic and diagnostic for hemochromatosis. It should be performed in patients with elevated ferritin and transferrin saturation. ${ }^{3}$ A patient's response to phlebotomy is diagnostic; patients with pathological iron overload will require multiple phlebotomies, whereas patients with other causes of elevated ferritin will show a rapid decrease in ferritin. ${ }^{3}$ The recommended schedule involves biweekly phlebotomy $(500 \mathrm{~mL})$, with measurement of hemoglobin and ferritin at each visit, continuing until the patient's ferritin level is less than $50 \mu \mathrm{g} / \mathrm{L}$. $^{3}$

\section{Case resolution}

Investigations for secondary causes of elevated ferritin (i.e., creatinine, erythrocyte sedimentation rate, hepatitis serology and ultrasonography of the liver) had normal results. Fasting glucose and glycated hemoglobin results suggested adequate glucose control while taking metformin. Mildy elevated triglycerides and reduced highdensity lipoprotein cholesterol were present; however, a waist circumference of $88 \mathrm{~cm}$ excluded a diagnosis of metabolic syndrome. A transferrin saturation of $60 \%$ suggested hemochromatosis, but genetic testing showed no copies of the C282Y allele and a single copy of the H63D allele. Despite these results, the patient was counselled that his clinical presentation was consistent with iron overload and arrangements were made for therapeutic phlebotomy, which would assist in confirming a diagnosis of hemochromatosis. In addition, the patient was counselled to stop consuming alcohol.

\section{References}

1. Pietrangelo A. Hereditary hemochromatosis - a new look at an old disease. N Engl J Med 2004;350:2383-97.

2. Camaschella C, Poggiali E. Towards explaining "unexplained hyperferritinemia." Haematologica 2009;94:307-9.

3. European Association for the Study of the Liver. EASL clinical practice guidelines for HFE hemochromatosis. J Hepatol 2010; 53:3-22.

4. Bacon BR, Adams PC, Kowdley KV, et al. Diagnosis and management of hemochromatosis: 2011 practice guideline by the American Association for the Study of Liver Diseases. Hepatology 2011;54:328-43.

5. Steinberg KK, Cogswell ME, Chang JC, et al. Prevalence of $\mathrm{C} 282 \mathrm{Y}$ and H63D mutations in the hemochromatosis (HFE) gene in the United States. JAMA 2001;285:2216-22.

Affiliations: Department of Medicine (Lanktree, Crowther) and Pathology (Sadikovic, Crowther), McMaster University, Hamilton, Ont.

Contributors: All of the authors contributed to the conception of the article and the fictitious patient. Matthew Lanktree drafted the manuscript. Bekim Sadikovic and Mark Crowther revised the manuscript for important intellectual content. All of the authors approved the final version submitted for publication.

Acknowledgements: The authors thank Dr. Bruce Lanktree and Dr. Guillaume Paré for their valuable contributions to the development of the fictitious patient and for providing feedback on the final manuscript.

Decisions is a series that focuses on practical evidence-based approaches to common presentations in primary care. The articles address key decisions that a clinician may encounter during initial assessment. The information presented can usually be covered in a typical primary care appointment. Articles should be no longer than 650 words, may include one box, figure or table and should begin with a very brief description (75 words or less) of the clinical situation. The decisions addressed should be presented in the form of questions. A box providing helpful resources for the patient or physician is encouraged. 